\title{
A Review on Glucocorticoids Induced Osteoporosis: Patho- genesis, Diagnosis and Management by Some Natural Plants
}

\author{
Amal K. Khaliefa ${ }^{1}$, Mohamed A. El-Desouky ${ }^{2}$, Walaa G. Hozayen ${ }^{1,3^{*}}$, Hanan A. Soliman ${ }^{1}$ \\ ${ }^{1}$ Faculty of Science, Biochemistry Division, Chemistry Department, Beni-suef University, Beni-Suef, Egypt \\ ${ }^{2}$ Faculty of Science, Biochemistry Division, Chemistry Department, Cairo University, Giza, Egypt \\ ${ }^{3}$ Faculty of Postgraduate Studies for Advanced Sciences, Biotechnology Department, Beni-Suef University, Beni-Suef, Egypt
}

*Corresponding author: Walaa G. Hozayen, Faculty of Science, Biochemistry Division, Chemistry Department. Faculty of Postgraduate Studies for Advanced Sciences, Biotechnology Department, Beni-suef University, Beni-Suef, Egypt, Tel: +201001068433; E-mail: Walaahozayen@hotmail.com

\begin{abstract}
200 million person all over the world affected by osteoporosis as it is most common metabolic bone disorder. The general reason of bone loss are age and menopause, and the rates of bone loss variable between individuals. Physical activity and environmental factors as nutrition can contribute to bone health as they modify both peak bone mass and following bone loss. Medications embrace both antiresorptive and anabolic types. Antiresorptive medications acts by reducing rates of bone remodeling like estrogens, selective estrogen receptor modulators (raloxifene), bisphosphonates (alendronate, risedronate, and ibandronate) and calcitonins. Parathyroid hormone is the only anabolic medication. The nonmedical treatment must be used with pharmacologic treatment to maximize outcomes for osteoporotic patient. Some plant-derived natural products, mostly phytoestrogens (isoflavones, lignans, coumestanes, stilbenes) and many more novel estrogen-like compounds in plants have been greatly used to prevent depletion in bone mineral density.

In this review an attempt has been made out to compile the mechanisms of glucocorticoids induced osteoporosis, the medicinal plants which are commonly utilized in osteoporosis with their prominent chemical ingredients and certain pharmacological actions.
\end{abstract}

Received Date: January 29, 2016

Accepted Date: February 04, 2016

Published Date: February 10, 2016

Citation: Hozayen, W.G., et al. A Review on Glucocorticoids Induced Osteoporosis: Pathogenesis, Diagnosis and Management by Some Natural Plants. (2016) Int J Food Nutr Sci 3(1): 204-209.

DOI: $10.15436 / 2377-0619.16 .047$

Keywords: Osteoporosis; Glucocorticoids; Biochemical markers; Natural plants

\section{Introduction}

Bone consists of cells (osteocytes, osteoblasts and osteoclasts), extracellular organic components (collagen and non-collagenous matrix protein) and non-organic components (calcium hydroxyapatite). The life span of bones needs balance between structural, functional and mechanical properties. Bone is continually remodeling itself by the action of osteocytes that stimulate osteoclasts activity which erode cavities in bone or inhibit the action of osteoblasts which synthesize a new bone matrix. The bone remodeling prevents fatigue damage and important in preserving calcium homeostasis and the loss of osteoblast - osteoclasts coupling lead to several metabolic bone diseases including osteoporosis, tumor related bone disease, rheumatoid arthritis ${ }^{[1,2]}$.

Genetic factors (e.g. genes regulating bone mass are vitamin D receptor gene, estrogen receptor gene, transforming growth factor B, interleukin-6 and gene encoding type 1 collagen) and environmental factors (e.g. exercise, nutritional status, drugs like contraceptive pills and glucocorticoids, neoplastic diseases such as myeloma, leukemia, other causes like lactation, parity, alcohol, smoking, weight loss and long hip axis length) are the major causes of peak bone mass ${ }^{[3]}$. 
The term "osteoporosis" exactly means "porous bone" and it results from an abnormal bone remodeling process because bone formation and/or the mechanical response to physical strain are not sufficient to produce adequate fracture resistance. Osteoporosis is a syndrome of dysadaptation which leads to low bone mass, bone microarchitecture disruption of bone tissue, decreased skeletal strength and increased predisposition to fracture at different sites as the spine, hip or wrist ${ }^{[4,5]}$.

The International Osteoporosis Foundation (IOF) reported that $30-50 \%$ of women and $15-30 \%$ of men will be plagued in their lifespan ${ }^{[6]}$. Also IOF reported that Egyptian women have lower bone mineral density compared to western women and $28.4 \%$ of postmenopausal women in Egypt are expected to have osteoporosis ${ }^{[7]}$. It noted that the incidence of fracture in male low than female due to men acquire a greater bone density than women as their body weight and physical activity are higher and androgen concentration increases bone density to higher levels than estrogen $\mathrm{do}^{[8]}$.

Osteoporosis had been classified into primary and secondary, revealing themselves through specific signs and symptoms ${ }^{[9]}$. Osteoporosis is considered primary when it follows natural menopause (postmenopausal osteoporosis) or develops in the normal course of aging (senile osteoporosis) and is characterized by low bone mass in the absence of a disease process or exposure ${ }^{[10]}$. In Type 1 osteoporosis, cortical bone was less affected than trabecular bone, leading to fractures in regions high in trabecular bone content, the spine and distal forearm ${ }^{[11]}$. Secondary osteoporosis is associated with a known triggering disease or medications ${ }^{[12]}$ that listed in Table 1.

Table 1: Causes of Secondary Osteoporosis

\begin{tabular}{|c|c|c|c|}
\hline $\begin{array}{l}\text { Life Style } \\
\text { • Excess } \\
\text { alcohol } \\
\text { - Smoking } \\
\text { - Physical } \\
\text { inactivity } \\
\text { - Low body } \\
\text { weight }\end{array}$ & $\begin{array}{l}\text { Drugs } \\
\text { - Glucocorti- } \\
\text { coids } \\
\text { - Antiepileptic } \\
\text { - Anticoagu- } \\
\text { lant (Heparin) }\end{array}$ & $\begin{array}{l}\text { Endocrine } \\
\text { diseases } \\
\text { • Hypogonad- } \\
\text { ism } \\
\text { - Hyperthy- } \\
\text { roidism } \\
\text { - Cushing's } \\
\text { disease } \\
\text { - Growth } \\
\text { hormone } \\
\text { deficiency } \\
\text { - Hyperpara- } \\
\text { thyroidism }\end{array}$ & $\begin{array}{l}\text { Medical disorders } \\
\text { - Malnutrition or } \\
\text { malabsorption } \\
\text { - Anorexia nervosa } \\
\text { - Inflammatory } \\
\text { intestinal disease } \\
\text { - Intestinal resection } \\
\text { - Chronic inflamma- } \\
\text { tory disease (rheu- } \\
\text { matoid arthritis). } \\
\text { - Transplant patients } \\
\text { (solid organs and } \\
\text { bone marrow) } \\
\text { - Systemic masto- } \\
\text { cytosis }\end{array}$ \\
\hline
\end{tabular}

\section{Glucocorticoids induced osteoporosis (GIO):}

Endogenous glucocorticoids are hormones released by the adrenal cortex in response to stress. Steroids are modulated by the hypothalamic- pituitary-adrenal axis to release corticotropin-releasing hormone, which acts on the anterior pituitary to stimulate the synthesis and secretion of adrenocorticotropic hormone $(\mathrm{ACTH})$ which in turn stimulates the adrenal cortex to produce glucocorticoids ${ }^{[13,14]}$.

Exogenous glucocorticoids (GC) are synthetic compounds [dexamethasone (Dex) and methyl prednisolone (MP)] used for the treatment of autoimmune diseases, rheumatism, gastrointestinal diseases, organ transplantation and to treat cancers of the lymphoid system, such as leukemias, lymphomas, and myelomas ${ }^{[13,15]}$ but it produced many side effects by longterm use such as hyperglycemia, weight gain, osteoporosis, depression, decreased immunological function and glucocorticoid-induced osteoporosis (GIO) which is the most serious side effects $^{[16]}$

GIO is biphasic, loss of bone mineral density is known to be rapid $8-12 \%$ during first year because of excessive rate of bone resorption, followed by a slower annual loss of approximately $2-4 \%$. The harmful effect of excess GCs on the skeleton might be on bone cells which is direct effect or on extra skeletal tissues or chemical mediators that is indirect effects ${ }^{[17]}$.

Direct effects involve activation of monomeric glucocorticoid receptor signaling participates in excessive bone remodeling, it impairs osteoblast survival and differentiation, inhibit the production of bone matrix components, increase osteoblastic and osteocytic apoptosis, enhanced secretion of the osteoclast-promoting factor receptor activator of nuclear factor kappa B ligand (RANKL) by osteogenic cells, activation of osteoclastic cells and diminishing absorption of calcium. Indirect effects include suppress endogenous gonadal steroids and reduced of muscle volume and strength ${ }^{[18,19]}$.

\section{Effect of glucocorticoids on osteoblasts}

The hypo function and apoptosis of osteoblasts is the first action of GC-induced bone loss as GCs induce a 30\% increase in apoptosis of osteoblast and osteocyte ${ }^{[20]}$. The decreasing number and function of osteoblastic lead to decreasing osteoblastogenesis and the pool of preosteoblastic cells that can differentiate into mature osteoblasts ${ }^{[21]}$.

GCs defeat osteoblast function is associated with Wnt signaling pathway alteration which is a critical pathway for differentiation of mesenchymal cells toward mature osteoblasts leading to reduction of osteoblastogenesis. GCs enhance Dickkopf 1 expression, one of the Wnt antagonists that prevents soluble Wnt proteins from binding to their receptor complex. GCs maintain levels of glycogen-synthase kinase- $3 \beta$, a key kinase that phosphorylates $\beta$-catenin, thereby preventing the translocation of $\beta$-catenin into the nucleus and the initiation of transcription in favor of osteoblastogenesis ${ }^{[2]}$.

Glucocorticoids differentiate bone marrow stromal cells to adipocytes instead of the osteoblastic linage. As osteoblastic function is decreased by glucocorticoids, the type I collagen synthesis is inhibited. In addition, the synthesis of insulin-like growth factor-1 (IGF-1), an agent that enhances bone formation, is suppressed by glucocorticoids ${ }^{[23]}$.

\section{Effect of glucocorticoids on osteoclasts}

Osteoclasts (bone resorbing cells) are derived from hematopoietic precursors and differentiated from osteoclast precursor to fully activated multinucleated osteoclast depends on receptor activator of NF- $\mathrm{kB}$ ligand (RANKL) which is essential for the formation, function, and survival of the osteoclasts and is a member of the tumor necrosis factor (TNF) family ${ }^{[24]}$.

Glucocorticoids act directly on osteoclasts to decrease the apoptosis of mature osteoclasts and increase osteoclastic bone resorption through an increase in receptor activator of NF$\kappa \mathrm{B}$ ligand (a RANKL) and a reduction in osteoprotegrin that is physiological inhibitor of RANKL ${ }^{[21]}$. As a result, there is an early increase in osteoclastogenesis, and a prolongation of the lifespan of osteoclasts has been implied in the pathogenesis of $\mathrm{GIO}^{[23]}$. 


\section{Effects of glucocorticoids on calcium}

Glucocorticoids influence bone health through interactions with Ca-vitamin D-PTH axis. Glucocorticoids decrease the calcium concentration via two main mechanisms. First, glucocorticoids reduce intestinal calcium absorption by opposing the action of vitamin D and decreasing the expression of calcium channels in the duodenum. Second, glucocorticoids increase renal calcium excretion by decreasing calcium reabsorption which leads to secondary hyperparathyroidism ${ }^{[25]}$.

\section{Diagnosis of osteoporosis by biochemical markers}

The general laboratory evaluation are performed to do a differential diagnosis of bone fragility includes complete blood cell count and calcium, phosphorous, alkaline phosphatase, thyrotropin, renal and liver function, 25-hydroxyvitamin D and urinary calcium excretion ${ }^{[26]}$.

Table 2 reveal the bone resorption markers determined in serum and the markers of bone formation measured ${ }^{[27]}$. Bone resorption markers, as 5b isoenzyme of tartrate resistant acid phosphatase (TRACP 5b), type I collagen crosslinks pyridinoline (PYD), deoxypyridinoline (DPD), the related telopeptides (N-terminal crosslinking telopeptides of type I collagen (NTX-I) and C-terminal crosslinking telopeptides of type I collagen (CTX-I). While bone formation markers as serum osteocalcin, bone-specific alkaline phosphatase (B-ALP) and procollagen type I N-terminal propeptide (PINP) ${ }^{[28]}$.

Table 2: Biochemical markers of bone turnover

\begin{tabular}{|l|l|}
\hline Bone formation markers: & $\begin{array}{l}\text { Bone Resorption markers: } \\
\text { - Osteocalcin }\end{array}$ \\
- Bone-specific alkaline & - Hydroxyproline \\
phosphatase & - Pyridinoline \\
- Procollagen Type I C & - Deoxypyridinoline \\
Propeptides (PICP) & • N-terminal cross-linking telopeptides \\
- Procollagen Type I N & of type I collagen (NTX-I) \\
Propeptides (PINP) & - C-terminal cross-linking telopeptides \\
& of type I collagen (CTX-I) \\
& - Procollagen Type I C Propeptides \\
& (PICP) \\
& - Tartrate-resistant acid phosphatase \\
\hline
\end{tabular}

\section{Diagnosis of osteoporosis by absorptiometry}

Osteoporosis is diagnosed clinically by measuring bone mineral density (BMD). Using dual-energy X-ray absorptiometry (DXA) to measures areal BMD (aBMD), whereas using quantitative computed tomography (QCT) allows measuring volumetric BMD (vBMD) of cortical and trabecular bone ${ }^{[29,30]}$.

Dual-energy X-ray absorptiometry (DXA) is based on the absorption of $\mathrm{X}$-rays which is very sensitive to the calcium content of tissue ${ }^{[8]}$. The most important determinant of fracture risk is low BMD. When BMD decreases to the cut-off of a T-score $<-2.5$ is index of osteopenia, osteoporosis, reduced bone strength and it gathers the WHO definition of osteoporo$\operatorname{sis}^{[31,32]}$.

Quantitative computerized tomography (QCT) scans can provide local assesses of cortical thickness, accurate cross-sectional geometric information and structural assesses of biomechanical significance as well as estimates bone strength to the lumbar spine hip, and peripheral sites $^{[33]}$. Unlike DEXA, QCT advancements enable precise and detailed assessments within the trabecular and cortical bone compartments. As a re- sult, it can make volume BMD determinations ${ }^{[34]}$.

\section{Management}

It is important to prevent early bone loss and to decrease in fracture risk as early as possible after the start of GC therapy $^{[17]}$. The goals of treatment are to prevent fracture, preserve structural bone integrity, Stop bone loss, preserve bone strength, and decrease morbidity and mortality related to fractures by decreasing factors contributing to fracture ${ }^{[35]}$. Besides drug treatments, non-pharmacological osteoporosis management is vital and very wide concept.

\section{Management by medications}

Osteoporosis therapies fall into two classes, antiresorptive drugs, which slow down bone resorption and anabolic drugs which stimulate bone formation ${ }^{[36]}$. Antiresorptive therapy include bisphosphonates (BP), selective estrogen receptor modulators (SERM) and calcitonin. Anabolic agents comprise PTH and its analogs ${ }^{[37]}$.

\section{Bisphosphonates}

Oral bisphosphonates (BPs) are first-line therapies and can be divided into non-nitrogen containing compounds (e.g. etidronate, clodronate, tiludronate) and nitrogen containing compounds (e.g. pamidronate, alendronate, ibandronate, risedronate and zoledronate). All BPs have a high affinity for calcium and in the body they concentrate in the skeleton at sites of active bone remodeling preventing fractures in osteoporotic patients and reduce the risk of fracture by up to $50 \% 0^{[38,39]}$.

\section{Selective estrogen receptor}

Selective estrogen receptor (SERMS) modulator treatment prevents postmenopausal bone loss and decreases the incidence of vertebral fracture ${ }^{[40]}$. Estrogens stimulate osteoblast differentiation and inhibit osteoclast proliferation and differentiation, resulting in increased bone formation. SERMs act differentially on the estrogen receptor (ER) in various tissues with a mixed agonist/antagonistic activity as estrogen receptor agonists in bone tissues while acting as estrogen receptor antagonists in the breast and uterus ${ }^{[41]}$.

\section{Calcitonin}

Calcitonin is hormone consist of a 32 amino acid and secreted by the C-cells of the thyroid gland. Secretion of calcitonin is stimulated by higher serum calcium concentration and gastrointestinal hormones such as gastrin. Calcitonin has antiresorptive effect by decreasing osteoclast activity leading to inhibit bone resorption ${ }^{[42]}$.

\section{Parathyroid hormone}

Parathyroid hormone (PTH) is a potent bone formation agent. It acts by increasing the osteoblast birth rate and improving the new bone formation. PTH have a favorable impact on BMD by stimulating bone formation rather than suppressing bone resorption ${ }^{[43]}$.

\section{Management by medicinal plants}

It turns out that previous studies show several dietary factors such as fruit, minerals and prebiotics can increase bone mineral density in osteoporotic patient ${ }^{[44]}$. Various types of 
medicinal plants approaches osteoporosis in different manner means some supply dietary calcium, some control the body's use of calcium, and some increase the level of certain hormones in the body ${ }^{[45]}$. Dark green vegetables are a viable source of dietary calcium and phytoestrogens (plant-derived compounds possessing estrogen- like activity) showed that they are promising alternatives to treat osteoporosis ${ }^{[2]}$.

\section{Flavonoids}

Flavonoids consist of large group of polyphenolic compounds and they have manifold beneficial biological effects owing to their antioxidant and estrogenic activities ${ }^{[46]}$. Flavonoids also called phytoestrogens because of their estrogenic activity with a chemical structure similar to $17 \beta$-estradiol naturally occurring estrogen bind to estrogen receptors and affecting estrogen-regulated processes ${ }^{[47]}$. Some flavonoids with potent antioxidant activity including scopoletin, resveratrol, and baicalein have found to exert anti-osteoporotic activities through suppressing osteoclast formation and tartrate-resistant acid phosphatase activity. So, phytoestrogens prevent bone resorption and increase bone density ${ }^{[48]}$.

Ocimum basilicum (Lamiaceae) generally known as sweet basil. Basil is herbaceous, perennial plant found in many regions of the world especially in the tropical regions of Africa, Asia and South and Central America and now cultivated worldwide under a variety of ecological conditions ${ }^{[49]}$.

Basil has shown numerous biological activities as nematicidal, an insecticidal, fungicidal, anti-inflammatory, hypolipidaemic, anti-platelet aggregation, antithrombotic, bronchodilatory and antiulcerogenic ${ }^{[50]}$. Many of medical effects of basil may be attributed to its antioxidant power due to flavonoids and polyphenols content like vanillic acid, lithospermic acid B, hydroxybenzoic acid, p-coumaric acid, syringic acid, ferulic acid, protocatechuic acid, caffeic acid, gentisic acid, tannins, linalool, eugenol, methyl chavicol, methyl cinnamate, ferulate, methyl eugenol, triterpenoids, steroidal glycoside ${ }^{[51]}$.

\section{Prebiotics}

Prebiotics not digested or absorbed in the small intestine but fermented in the large intestine by microbiota yielding short chain fatty acids and one of the short chain fatty acids is butyric acid that belongs to a new class of antiosteoporotic agents due to it increase calcium absorption in the large intestine and decrease bone loss. Several prebiotics such as inulin, fructo-oligosaccharides and galacto-oligosaccharide are thought to improve bone health ${ }^{[44]}$.

Cichorium intybus, also known as chicory and all parts of chicory plant possess medicinal importance due to it contains inulin, alkaloids, coumarins, sesquiterpene lactones, chlorophyll pigments, flavonoids, unsaturated sterols, saponins and tannins ${ }^{[52]}$. Moreover, chicory in rich with cichoric acid which stimulates the immune system as well as prevents inflammation and bacterial infections. So, it used as liver tonic, cardiotonic, diuretic, cholagogue, stomachic, emmenagogue, depurative, cephalalgia, hepatomegaly, inflammations, dyspepsia, anorexia, colic, flatulence, jaundice, amenorrhea dysmenorrhea, splenomegaly and asthma ${ }^{[3,54]}$.

Chicory leaves are good sources of anthocyanins, vitamins $\mathrm{A}$ and $\mathrm{C}$ in addition to calcium, phosphorus and inulin-type fructans that enhances the calcium uptake, enhance bone health by increasing bone mineral content (BMC) and bone mineral density (BMD) $)^{[55]}$.

\section{Apiaceae}

Petroselinum crispum commonly known as parsley which is biennial herb belongs to the family Apiaceae. Parsley cultivated in the Europe, Africa, Asia, central Mediterranean region, Southern Italy, Algeria and Tunisia ${ }^{[56]}$. Parsley is widely used in the medicinal and food industries because it contain phenolic compounds, flavonoids (apiin, Apigenin, luteolin, and apigenin-glycosides), ascorbic acid, tocopherol, volatile compounds (myristicin, apiole), coumarines (bergapten, imperatorin, phthalides, furanocoumarins, sesquiterpenes), vitamins, and nutrients ${ }^{[57,58]}$.

Parsley extract has been used for the treatment of osteoporosis as it contains both calcium and vitamin c, in addition to ergosterol (a precursor of vitamin D) which helps the body absorb and utilize the calcium. Also, parsley contain apigenin which has antitumor and antioxidant properties and some studies reported that apigenin inhibits osteoclastogenesis and osteoclast function. The extract from the aerial parts of parsley showed potent estrogenic activity which is equal to that of isoflavone glycosides from soybean ${ }^{[59]}$.

\section{Acknowledgement}

The authors are thankful to Faculty of Science, BeniSuef University for help in conducting this study and providing all required facilities.

\section{NO CONFLICT OF INTEREST}

\section{References}

1. Buehring, B., Viswanathan, R., Binkley, N., et al. Glucocorticoid-induced osteoporosis: An update on effects and management. (2013) J Allergy ClinImmunol 132(5): 1019-1030.

2. Lucinda, L.M., Aarestrupa, B.J., Brandão, J.S., et al. The effect of Ginkgo biloba extract treatment in the Bcl-2 expression by osteoblasts in the femoral trabecular bone of Wistar rats with glucocorticoid-induced osteoporosis. (2014) Rev Bras Farmacogn 24(3): 363-366.

3. Rath, K., Mohanty, B.B., Kumar, S., et al. Do statins have potential as anti-osteoporotic drugs and can they be used for prevention or targeting osteoporosis: A Review. (2013) Int J Curr Res Rev 5(4): 73-81.

4. Urano, T., Inoue, S. Genetics of osteoporosis. (2014) BiochemBiophys Res Commun 452(2): 287-293.

5. Unni, S., Yao, Y., Milne, N., et al. An evaluation of clinical risk factors for estimating fracture risk in postmenopausal osteoporosis using an electronic medical record database. (2015) OsteoporosInt 26(2): 581-587.

6. Tural, S., Kara, N., Alayli, G., et al. Association between osteoporosis and polymorphisms of the bone Gla protein, estrogen receptor 1, collagen 1-A1 and calcitonin receptor genes in Turkish postmenopausal women. (2013) Gene 515(1): 167-172.

7. Hossien, Y.E., Tork, H.M., El-Sabeely, A.A. Osteoporosis knowledge among female adolescents in Egypt. (2014) Am J Nurs 3(2): 13-17.

8. Hernlund, E., Svedbom, A., Ivergård, M., et al. Osteoporosis in the European Union: medical management, epidemiology and economic burden. (2013) Arch Osteoporos 8: 136.

9. Toti, P., Sbordone, C., Martuscelli, R., et al. Gene clustering analysis in human osteoporosis disease and modifications of the jawbone. (2013) Arch Oral Biol 58(8): 912-929.

10. Liang, W., Zhuo, X., Tang, Z., et al. Calcitonin gene-related peptide stimulates proliferation and osteogenic differentiation of osteoporotic 
rat-derived bone mesenchymal stem cells. (2015) Mol Cell Biochem 402(1-2): 101-110.

11. Adler, R.A. Laboratory testing for secondary osteoporosis evaluation. (2012) ClinBiochem 45(12): 894-900.

12. Martínez-Morillo, M., Grados, D., Holgado, S. Premenopausal Osteoporosis: How to Treat? (2012) ReumatolClin 8(2): 93-97.

13. Oakley, R.H., Cidlowski, J.A. The biology of the glucocorticoid receptor: New signaling mechanisms in health and disease. (2013) J Allergy ClinImmunol 132(5): 1033-1044.

14. Pan, G., Cao, J., Yang, N., et al. Role of Glucocorticoid-Induced Leucine Zipper (GILZ) in Bone Acquisition. (2014) J BiolChem 289(28): 19373-19382.

15. Lespessailles, E. Bisphosphonates and glucocorticoid-induced osteoporosis: Efficacy and tolerability. (2013) Joint Bone Spine 80(3): 258-264

16. Lin, S., Huang, J., Zheng, L., et al. Glucocorticoids - induced osteoporosis in Growing rats. (2014) Calcif Tissue Int 95(4): 362-373.

17. Suzuki, Y., Nawata, H., Soen, S., et al. Guidelines on the management and treatment of glucocorticoid-induced osteoporosis of the Japanese Society for Bone and Mineral Research: 2014 update. (2014) J Bone Miner Metab 32(4): 337-350.

18. Samir, S.M., Malek, H.A. Effect of cannabinoid receptors 1 modulation on osteoporosis in a rat model of different ages. (2014) J PhysiolPharmacol 65(5): 687-694.

19. Trementino, L., Ceccoli, L., Concettoni, C., et al. Fracture risk assessment before and after resolution of endogenous hypercortisolism: is the FRAX ${ }^{\circledR}$ algorithm useful? (2014) J Endocrinol Invest 37(10): 957-965.

20. Chen, F., Zhang, L., OuYang, Y., et al. Glucocorticoid Induced Osteoblast Apoptosis by Increasing E4BP4 Expression via Up-regulation of Bim. (2014) Calcif Tissue Int 94(6): 640-647.

21. Böcker, W., El Khassawna, T., Bauer, N., et al. Short-term glucocorticoid treatment causes spinal osteoporosis in ovariectomized rats. (2014) Eur Spine J 23(11): 2437-2448.

22. Yao, W., Dai, W., Jiang, J.X., et al. Glucocorticoids and osteocyte autophagy. (2013) Bone 54(2): 279-284.

23. Guañabens, N., Gifre, L., Peris, P. The role of Wnt signaling and sclerostin in the pathogenesis of glucocorticoid-induced osteoporosis. (2014) CurrOsteoporos Rep 12(1): 90-97.

24. Silva-Fernấndez, L., Rosario, M.P., Martínez-López, J.A., et al. Denosumab for the treatment of osteoporosis: A systematic literature review. (2013) ReumatolClin 9(1): 42-52.

25. Schiro, A., Stein, D.J. Why Can't I Just Stay on Prednisone? The Long-Term Adverse Effects of Steroids. (2015) Springer International Publishing 119-128.

26. Fisher, L., Fisher, A. Associations between liver Function, bone turnover biomarkers and adipokines in older patients with hip fracture. (2015) J EndocrinolMetabol 4(5-6): 121-135.

27. Eastell, R. Identification and management of osteoporosis in older adults. (2013) Medicine 41(1): 52-74.

28. Biver, E., Chopin, F., Coiffier, G., et al. Bone turnover markers for osteoporotic status assessment? A systematic review of their diagnosis value at baseline in osteoporosis. (2012) Joint Bone Spine 79(1): 20-25. 29. Laurent, M., Gielen, E., Claessens, F., et al. Osteoporosis in older men: Recent advances in pathophysiology and treatment. (2013) Best Pract Res ClinEndocrinolMetab 27(4): 527-539.

30. Liu, H.F., He, H.C., Yang, L., et al. Pulsed electromagnetic fields for postmenopausal osteoporosis and concomitant lumbar osteoarthritis in southwest China using proximal femur bone mineral density as the primary endpoint: study protocol for a randomized controlled trial. (2015) Trials 16(1): 265-271.

31. Yang, S., Center, J.R., Eisman, J.A., et al. Association between fat mass, lean mass, and bone loss: the Dubbo osteoporosis epidemiology study. (2015) OsteoporosInt 26(4): 1381-1386.

32. Noordin, S., Glowacki, J. Parathyroid hormone and its receptor gene polymorphisms: implications in osteoporosis and in fracture healing. (2016)RheumatolInt 36(1): 1-6.
33. Eriksen, E.F., Keaveny, T.M., Gallagher, E.R., et al. Literature review: The effects of teriparatide therapy at the hip in patients with osteoporosis. (2014) Bone 67: 246-256.

34. Mohammed, O.A., Shaheen, H.E., Kaoud, Y.E. Role of family medicine in the early detection and management of osteoporosis. (2014) Menoufia Med J 27(4): 833-839.

35. Küçükçakir, N., Altan, L., Korkmaz, N. Effects of Pilates exercises on pain, functional status and quality of life in women with postmenopausal osteoporosis. (2013) J BodywMovTher 17(2): 204-211.

36. Jin, S., Yan, Z., Tieyi, Y., et al. Eph-ephrin bidirectional signalling: A promising approach for osteoporosis treatment. (2013) J Med Hypotheses Ideas 7(2): 40-42.

37. Miladi, K., Sfar, S., Fessi, H., et al. Drug carriers in osteoporosis: Preparation, drug encapsulation and applications. (2013) Int J Pharm 445(1-2): 181-195.

38. Hawley, S., Javaid, M.K., Rubin, K.H., et al. Incidence and predictors of multiple fractures despite high adherence to oral bisphosphonates: A Binational Population-Based Cohort Study. (2016) J Bone Miner Res 31(1): 234-244.

39. Kates, S.L., Ackert-Bicknell, C.L. How do bisphosphonates affect fracture healing? (2016) Injury 47(Suppl 1): S65-68.

40. Park, S.B., Kim, C.H., Hong, M., et al. Effect of a selective estrogen receptor modulator on bone formation in osteoporotic spine fusion using an ovariectomized rat model. (2016) Spine J 16(1): 72-81.

41. Ouellet, C., Ouellet, É., Poirier, D. In vitro evaluation of a tetrahydroisoquinoline derivative as a steroid sulfatase inhibitor and a selective estrogen receptor modulator. (2015) Invest New Drugs 33(1): 95-103. 42. Felsenfeld, A.J., Levine, B.S. Calcitonin, the forgotten hormone: does it deserve to be forgotten? (2015) Clin Kidney J 8(2): 180-187.

43. Zhang, Q., Qian, J., Zhu, Y. Parathyroid hormone plus alendronate in osteoporosis: a meta-analysis of randomized controlled trials. (2015) Int J ClinExp Med 8(3): 3338-3348.

44. Yang, L., Lu, T., Lin, W. The prebiotic arabinogalactan of Anoectochilusformosanus prevents ovariectomy-induced osteoporosis in mice. (2013) J Funct Foods 5(4): 1642-1653.

45. Rajagopal, P.L., Amrutha, C., Premaletha, K., et al. Medicinal Plants in Osteoporosis- A Review. (2013) Int J Adv Pharm BiolChem 2(4): 605-608.

46. Xu, F., Ding, Y., Guo, Y., et al. Anti-osteoporosis effect of Epimedium via an estrogen-like mechanism based on a system-level approach. (2016) J Ethnopharmacol 177:148-160.

47. Horcajada, M.N., Offord, E. Naturally plant-derived compounds: role in bone anabolism. (2012) CurrMolPharmacol 5(2): 205-218.

48. Morgan, A.M., Lee, H.W., Lee, S., et al. Anti-osteoporotic and antioxidant activities of chemical constituents of the aerial parts of Ducrosiaismaelis. (2014) Bioorg Med ChemLett 24(15): 3434-3439.

49. Siddiqui, B.S., Bhatti, H.A., Begum, S., et al. Evaluation of the antimycobacterium activity of the constituents from Ocimumbasilicum against Mycobacterium tuberculosis. (2012) J Ethnopharmacol 144(1): 220-222.

50. Ibrahim, M.M., Al-Ansary, A.M., Abd-El-Hamid, A.M., et al. Impact of location and seasonal variability on the herbage and essential oil yields of two grown sweet basil varieties in Egypt. (2013) Aust J Basic ApplSci 7(1): 441-447.

51. Bora, K.S., Arora, S., Shri, R. Role of Ocimumbasilicum L. in prevention of ischemia and reperfusion-induced cerebral damage, and motor dysfunctions in mice brain. (2011) J Ethnopharmacol 137(3): 13601365.

52. Abbas, Z.K., Saggu, S., Sakeran, M.I., et al. Phytochemical, antioxidant and mineral composition of hydroalcoholic extract of chicory (Cichoriumintybus L.) leaves. (2015) Saudi J BiolSci 22(3): 322-326.

53. Ahmed, O.M., Hozayen, W.G., Bastawy, M., et al. Biochemical Effects of Cichoriumintybus and Sonchusoleraceus Infusions and Esculetin on Streptozotocin-Induced Diabetic Albino Rats. (2011) J Am Sci 7(7): 1124-1137.

54. Al-Malki, A.L., Abo-Golayel, M.K. Hepatoprotective Efficacy of Chicory alone or combined with Dandelion leaves against induced Liv- 
er Damage. (2013) Life Sci J 10(4): 140-157.

55. Weisstaub, A.R., Abdala, V., Gonzales, C.M., et al. Polydextrose Enhances Calcium Absorption and Bone Retention in Ovariectomized Rats. (2013) Int J Food Sci 8.

56. Tang, E.L., Rajarajeswaran, J., Fung, S., et al. Petroselinumcrispum has antioxidant properties, protects against DNA damage and inhibits proliferation and migration of cancer cells. (2015) J Sci Food Agri 95(13): 2763-2771.

57. Li, M.Y., Tan, H.W., Wang, F., et al. De Novo Transcriptome Sequence Assembly and Identification of AP2/ERF Transcription Factor Related to Abiotic Stress in Parsley (Petroselinumcrispum). (2014) PloS One 9(9): e108977.

58. Hozayen, W.G., Hegab, M.Y., Soliman, H.A. Effects of parsley and pumpkin on alcohol induced testicular damage in rat model. (2015) J IntAcad Res Multidiscip 2(12):446-455.

59. Goto, T., Hagiwara, K., Shirai, N., et al. Apigenin inhibits osteoblastogenesis and osteoclastogenesis and prevents bone loss in ovariectomized mice. (2015) Cytotechnology 67(2): 357-365. 